Journal of Energy and
Environmental Sulficial publication of the International Society for Energy, Environment and Sustainability (ISEES)
Journal homepage : www.jees.in

\title{
Parametric Study of Intrinsic Thermoacoustic Feedback-driven Instability in a Partially Premixed Combustor
}

\author{
Manmohan Vishwakarma*1, Sathesh Mariappan² \\ ${ }^{*}$ Advanced Combustion and Acoustic Laboratory, Department of Aerospace Engineering, Indian Institute of Technology Kanpur \\ ${ }^{2}$ Associate Professor, Advanced Combustion and Acoustic Laboratory, Department of Aerospace Engineering, Indian Institute of Technology Kanpur
}

\section{A R T I C L E I N F O}

Received : 20 June 2019

Revised : 28 August 2019

Accepted : 04 October 2019

Keywords:

Thermo acoustic instability;

partially premix swirl combustor;

Degree of premixing:

Intrinsic thermo acoustic feedback

\begin{abstract}
A B S T R A C T
The presence of turbine blades in aero engines effectively reduces the exit area of the combustor. In present study, experiments are performed on reduced area at the exit of combustor by keeping a compact nozzle. Reducing the exit area leads to intrinsic thermo acoustic (ITA) mode of instability inside the combustor. A parametric study is performed to understand the characteristics of ITA feedbackdriven instability in a partially premixed, swirl stabilized turbulent combustor. ITA driven instability occurs in combustors, where significant acoustic losses are present. In the absence of nozzle, conventional acoustic-driven instability is observed. On the other hand, when the nozzle is partially closed end (12\% of the combustor cross-section area), significant acoustic losses occur, leading to the formation of ITA driven instability. In the present study, a wide range of parameters such as area at exit, length of the combustor, location of fuel injection are varied to understand the variation of frequency and mode shapes associated with acoustic and ITA driven instabilities. The area at the exit play role in controlling the acoustic energy losses. The location of fuel injection plays a vital role in thermo acoustic instability. The mixing length is varied from $80 \mathrm{~mm}$ to $40 \mathrm{~mm}$. In both cases, the degree of premixing is varied, which is directly proportional to the residence time of fuel and air in the mixing tube. The degree of premixing can be an important factor for the study of ITA mode.

(C) 2020 ISEES, All rights reserved
\end{abstract}

\section{Introduction}

The demand for low $\mathrm{NO}_{\mathrm{x}}$ emission leads to the burning of fuel at lean conditions, which is susceptible to combustion instability in the form of combustion induced pressure oscillations (Venkataraman et al. 1999). Combustion instability is a significant problem, which may lead to gradual degradation or sudden failure in aero and land-based gas turbine engines. For the combustion instability to occur inside the combustion chamber, Rayleigh postulated that if the pressure oscillation and the heat release oscillations are in the same phase, the pressure oscillations are amplified which lead to instability inside the combustion chamber (Strutt and Baron Rayleigh 1877).

Generally, the combustion instability is classified in three categories based on interaction within the components involved: 1) chamber instabilities, 2) intrinsic instabilities, and 3) system instabilities(Williams 1985). The present study is concerned with the system instabilities of the combustor. System instability is mainly concerned with the interaction of combustion to its surrounding medium, which can be mainly acoustically driven instability (Duran et al. 2014), entropy driven instability (Morgans and Duran 2016) and intrinsic thermo acoustic (ITA) instability (Hoeijmakers et al. 2016).

The acoustically driven instability is caused due to the feedback between unsteady flame and the acoustic waves. An unsteady flame/ unsteady heat source is an efficient source of acoustic waves. The generated waves are reflected from the boundaries of the combustion chamber and give rise to the unsteady flow field inside the combustor. When the unsteady flow field interacts with the flame front, the flame responds with unsteady heat release rate, either by locally altering the equivalence ratio or through hydrodynamic instabilities. The dominant frequency of the feedback mechanism of instability is a strong function of the acoustic lengthscale (in general, the axial length) of the combustor and speed of sound (Tim Lieuwen 2003).

The perturbation of equivalence ratio $(\phi)$ also plays a critical role in instability mechanism inside the combustor (Venkataraman et al. 1999). The hot spots created due to fluctuating equivalence ratio travel downstream of the combustor where these hot spots encounter area restrictions or area change which leads to generation of acoustic waves. These acoustic waves reflect and disturb the flame which gradually evolve into entropy driven instability (Morgans, Goh, and Dahan 2013) (Marble and Candel 1977).

The acoustic losses are linked to the reflection coefficient at the exit boundary condition of the combustor. The exit area of the gas turbine combustor changes due to the presence of turbine blades at the exit, which reduces the reflection coefficient. Much of the acoustic waves are not reflected, which results in a weak feedback from the exit, damping

\footnotetext{
*Corresponding Author: :mohanv@iitk.ac.in
} 
acoustic driven instability. On the other hand, since the flame acts as an acoustic source, acoustic waves propagate upstream as well as downstream of the flame. The waves propagating upstream of the flame perturb the incoming reactant mixture, which again disturbs the flame leading to a feedback loop resulting in ITA driven instability (Hoeijmakers et al. 2015).

The present literature survey deals with effect of 1) flame dynamics 2) boundary conditions and 3) different geometrical parameters on feedback mechanism, mainly on ITA mode. The survey mentions first on the numerical study and later on the experimental investigations.

In the numerical study,Tay-Wo-Chong et al. (Tay-Wo-Chong et al. 2011) presented an idea of three modes which were identified as Helmholtz mode between plenum and combustion chamber, quarter wave mode due to acoustic resonance and flame dynamics mode which was independent from the feedback loop. They also compared the results for numerical and experimental study and stated that even a slight variation of predicted Flame transfer function (FTF) has significant effect onthe growth rate of instability. FTF is defined as the ratio of normalized heat release rate fluctuations to the normalized velocity fluctuations [12] (Durox et al. 2009). Flame dynamics mode has representative characteristics of ITA mode. The idea of ITA mode was first mentioned in Hoeijmakers et al.(Hoeijmakers et al. 2014) and Courtine et al.,(Courtine et al. 2014).

Hoeijmakers et al. (Hoeijmakers et al. 2014) modelled thermoacoustic interaction in a burner as a scattering matrix. It also states that at higher acoustic losses, the thermoacoustic system may be unstable. It may be due the fact that the flame acoustic coupling itself can be unstable. The stability of the burner was determined by the scattering matrix in the limit of zero acoustic reflection coefficient. The results were compared to the FEM model setup in Comsol Multiphysics and generated the same result as network model, which provided the evidence for intrinsic flame mode. The study also revealed that the flame can act as acoustic source and produce acoustic pressure and velocity when triggered by velocity fluctuations and thus forming a local feedback loop.

The FTF was related to ITA mode of instability in the numerical study of Courtine et al.(Courtine et al. 2014) by using $\eta-T$ model. The causality of Crocco's model for flame acoustic coupling leads to ITA mode of instability in anechoic environment. The DNS study revealed that the causality issue was due to the mode conversion present at the plane of inlet duct and the combustion chamber. It also stated that validity of Crocco's model gives that intrinsic thermoacoustic modes were existing. The ITA modes were controlled by mode conversion and kinematic over restoration of flame.

In the subsequent work,Hoeijmakers et al. (Hoeijmakers et al. 2015), identified two different scattering matrices for 1) active elements e.g., burner and 2) passive elements e.g., reflection coefficient at boundary. They show that increase in acoustic losses leads to stable flame inside the combustor, only for acoustic-driven instability, but not for ITA mode instability. Emmert et al. (Emmert, Bomberg, and Polifke 2015), identified that the mechanism involved was ITA mode. They also modelled the FTF from ç-ô model. It was found that in the limit of reflection coefficient reaching zero, eigenmodes were observed and these eigenmodes were identified as ITA modes.

Courtine et al.(Courtine, Selle, and Poinsot 2015) performed a direct numerical simulation (DNS) in variable area combustor with two dimensional Bunsen flame. They showed the presence of unstable ITA mode when the boundary conditions are anechoic. The simulations were performed with various area ratios (ratio of injection tube over combustion chamber) and lengths of combustor. It was founded that the frequency of ITA mode instability was independent of combustor acoustic field and only depends on FTF. It was also observed that with increase in acoustic energy loss, the system will become more unstable. Further they have explained the underlying physical mechanism using mode conversion and kinematic over restoration in the vicinity of flame.

Emmert et al. (Emmert et al. 2017) showed that in order to predict the stability of a thermoacoustic system, eigenmodes comprising both acoustic and ITA modes should be considered. Mukherjee et al. (Mukherjee and Shrira 2017) used dispersion equation to identify the mechanism involved in combustion instability. The dispersion equation was derived from the conservation equations applied on flame and system. The frequency (eigenvalue) identified from the solution of dispersion relation was a complex number. The real part of frequency revealed the oscillating frequency of the system and the imaginary part revealed the stability of the system. The study states that an eigenmode can be ITA or acoustic mode of instability inside the combustor, depends on the factor $\eta$ and $T$.

Silva et al.(Silva et al. 2017) performed a Large eddy simulation (LES), where they studied interaction of combustion instability and noise. The global heat release rate was expressed in terms of flame response and the combustion noise source which was utilised in network model to understand the influence of ITA feedback on combustion generated noise in a turbulent combustion. The LES study also revealed that the peaks in the spectral distribution of sound pressure level were found from resonances with acoustic cavity modes or intrinsic thermoacoustic (ITA) modes and not from corresponding peaks in the spectral distribution of the combustion noise source term.

Hosseini et al. (Hosseini et al. 2018) performed a numerical study, which revealed the behaviour of ITA mode for different cases of time delay, temperature ratio and reflection coefficient, covering up a broad range of parameter. The study uses Emmert et al. (Emmert et al. 2015) network model to identify the acoustic and ITA mode inside the combustor.

Chen et al.(Chen, Ayton, and Zhao 2019) performed a numerical study, where they used the anechoic boundary condition to identify ITA mode. Chen et al. proposed that the flame transfer function was affected by the flame front fluctuations, which were responsible for the entropy perturbation in combustor in the absence of equivalence ratio perturbation. The study considers the entropy and acoustic mode in model to determine the thermoacoustic oscillation where the ITA and chamberoriented feedbacks were included. The study confirms the result of Hoeijmakers et al. (Hoeijmakers et al. 2016) that the reflection coefficient can alter the ITA mode and cavity induced oscillations.

A few experimental studies also revealed the presence of ITA driven instability inside the combustor. ITA mode was identified experimentally by Hoeijmakers et al. (Hoeijmakers et al. 2016). Their experimental setup consists of a combustor (supporting planar acoustic waves), terminating in a horn, which provides an anechoic boundary condition. The horn cut off frequency was $150 \mathrm{~Hz}$, which was within the range of ITA mode frequency. The study also gives an experimental evidence for the flame fluctuation in higher acoustic losses at the exit boundary, which was already identified by many numerical studies.

In experimental and analytical study by Albyrak et al. (Albayrak et al. 2017) the swirler position, bulk flow velocity and length of combustor were varied. It was found out that ITA mode was insensitive to the effect of length of combustor. The bulk flow velocity and swirler position plays significant role on ITA mode. The experimental results were compared to LES and analytical study performed. The stability of ITA mode of instability depends on the gain and phase of the flame transfer function. It also stated that with the reflection coefficient tending to zero the ITA mode of instability exist. Murugesan et al.(Murugesan et al. 2018) performed experimental study on partially premixed flame. In experimenta study injection location, area ratio and length of combustor were varied.

From the above survey we observed that a few experimental investigations (Hoeijmakers et al. 2016) (Albayrak et al. 2017) (Murugesan et al. 2018) have been performed to show the occurrence of ITA mode driven instability in a range of operating conditions. The present work is mainly an experimental work on academic scale test rig. In this paper,we perform a detailed study on the modes of instability found in combustors: acoustic and ITA modes. The characteristics of these two modes vary differently with the length of the combustor. In order to identify their existence, an alteration of the length is required. Therefore extension ducts are used.

In a real gas turbine engine, the combustor is followed by turbine blades, which lead to a reduction in the exit area of the combustor. In our earlier investigation (Murugesan et al. 2018), it was observed that a reduction in the exit area was accompanied by a drop in the acoustic reflection coefficient; as a consequence, the conventional acoustic-driven instability is damped. However, the ITA mode becomes dominant as we reduce the exit area. With reduction in area at exit, the exit acoustic reflection coefficient is altered. In order to represent this scenario, we perform experiments in the combustion chamber, with the variable area at the exit $(A R)$ and varying the injection location in the mixing tube $\left(l_{\text {mix }}\right)$ and length of duct $(l)$ (figure 1 and 2 ). Many studies related to the identification of ITA mode discuss the effect of exit boundary condition on the flame (Murugesan et al. 2018) (Hoeijmakers et al. 2015) (Emmert et al. 2015). In a lean premix combustor, the degree of premixing plays a very vital role in identifying the $\mathrm{NO}_{\mathrm{x}}$ from the combustion (Hase and Kori 1996), but no study has been performed to identify the effect of degree of premixing on instability. The degree of premixing is a direct function of the residence time (Hase and Kori 1996) of fuel in the mixing tube which can be related to mixing length or the injection location in the mixing tube $\left(l_{\text {mix }}\right)$. The degree of premixing has significance relevance as the spatial and temporal fluctuations in equivalence ratio can cause thermoacoustic perturbations (Stopper et al. 2013). Data are compared for different operating conditions. This study provides an initial input on the effect $A R, l$ and $l_{m i x}$ on the flame instability. The present work is similar 
and an extension of the study performed by Murugesan et al.(Murugesan et al. 2018), where the characteristics of ITA driven instability are explored with parameters of area ratio $(A R)$, length of combustor $(l)$ and injection location $\left(l_{\text {min }}\right)$.

The present study is organized as follows; the experimental set up is discussed in detail in section 2 . In section 3 , results obtained for partially closed and open-end are discussed. Sections 4 describes about the main finding of the present study with future aspects of study.

\section{Methodology}

\subsection{Experimental details}

Experiments are performed in a swirl-stabilized turbulent partially premixed combustor (figure 1). It consists of a settling chamber, mixing tube, burner and combustion chamber with three extension ducts. Disturbances present in air feed lines are damped in the settling chamber. The diameter and length of the settling chamber are $220 \mathrm{~mm}$ and $260 \mathrm{~mm}$ respectively. The mixing tube length is $80 \mathrm{~mm}$. The burner consists of fuel injection system and swirler. Fuel is injected into the mixing tube circumferentially at four locations through a hole of $1 \mathrm{~mm}$ diameter at an axial location, which is varied between 40 and $80 \mathrm{~mm}$ (marked as $l_{\text {mix }}$ ) upstream of the swirler. The $l$ is measured from the entrance plane of combustor.The swirler diameter is $42 \mathrm{~mm}$ with 12 vanes at a vane angle of 30 degrees with a thickness of $10 \mathrm{~mm}$. The face of the swirler is flushmounted with the entrance plane of the combustion chamber. The length of the combustion chamber is $300 \mathrm{~mm}$. The different lengths of set up are marked as $l$ and $l$ in figure 1 .

Extension ducts are attached to the combustion chamber to achieve a total length (marked as $l$ ) of up to $1 \mathrm{~m}$. The length of the primary combustion chamber is 300 mmwith a cross section area of $90 * 90 \mathrm{~mm}$. There are three extension ducts of lengths $200 \mathrm{~mm}, 200 \mathrm{~mm}$, and $300 \mathrm{~mm}$, respectively. By using them, the total length $(l)$ of the combustor is varied to $0.7 \mathrm{~m}, 0.8 \mathrm{~m}$, and $1 \mathrm{~m}$. In this paper, experiments are performed at three lengths $l(1 \mathrm{~m}, 0.8 \mathrm{~m}$, and $0.7 \mathrm{~m})$. Similarly, the mixing length $\left(l_{\text {mix }}\right.$, the distance between the location of fuel injection and entrance plane of combustor) is also varied. The reduction in the exit area is achieved by placing a compact ( $10 \mathrm{~mm}$ axial length) removable nozzle (shown in figure 2). In the present study, the nozzle valve is partially closed, such that the area ratio $(A R=$ exit area/cross-section of combustor) is kept at 0.12 . The results are compared for different cases of experiments.

Liquefied petroleum gas (LPG) is used as fuel in present experimental analysis, which is available in India. Fuel mixes with air in the mixing tube. A spark plug is used to ignite the incoming mixture. Air and LPG flow rates are measured using rotameter calibrated with an uncertainty of $\pm 34 \mathrm{slpm}$ and $\pm 1.2 \mathrm{slpm}$ for air and fuel flow rate, respectively. Two different pressure regulators are used to supply the air and fuel in the feed line at a constant pressure of 4 and 2.5 bar. Table 1 gives the details for the location of different pressure transducers and thermocouple. The pressure transducer sensitivity is $215.8 \mathrm{kPa} / \mathrm{mV}, 222.2 \mathrm{kPa} / \mathrm{mV}$ and $218.5 \mathrm{kPa} / \mathrm{mV}$ respectively. Transducers are mounted on the combustor wall using a specially made stainless steel port. A K-type thermocouple is located at $0.45 \mathrm{~m}$ downstream of the swirler (shown in figure 1 symbol $\mathrm{T}$ ), is used to monitor the steady-state temperature. Unsteady pressure fluctuations are measured at a sampling rate of $1024 \mathrm{~Hz}$ for $4 \mathrm{~s}$. A semiinfinite tube (6 m long) is connected to the pressure transducer mounted to prevent the occurrence of acoustic resonance within the mount.

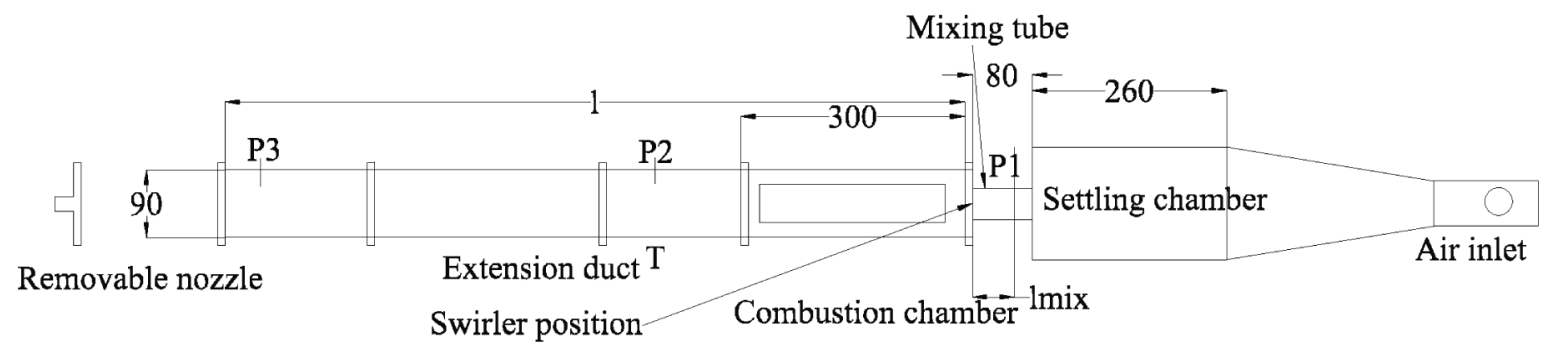

Figure 1: Schematic of the experimental setup, showing the major components.

All dimensions are in $\mathrm{mm}$. Details of the removable nozzle is shown in figure 2.

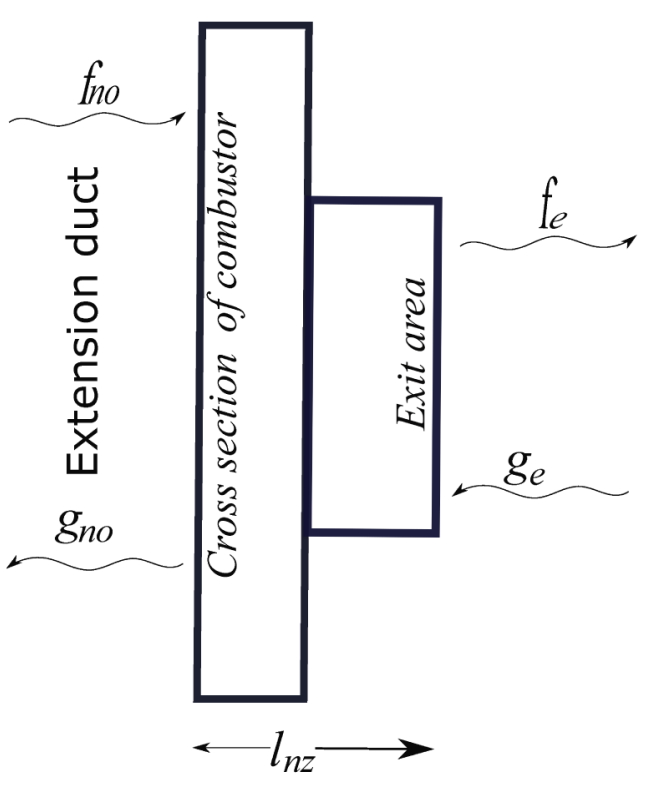

Figure2: Geometry of our variable area removable nozzle. The system is of length $l_{n z}$, a variable area nozzle beginning at $\tilde{x}_{n o}$ ending at $\widetilde{x}_{e}$. The conditionsimposed at the inlet and exit is also shown. The same has a thickness of $l_{n z} \ll \lambda$ along theaxial direction.
Table 1: Different pressure sensor location for different L, the US stands for upstream and DS stands for the downstream location from swirler

\begin{tabular}{c|c|c|c|c}
\hline $\begin{array}{c}\text { Pressure transducer } \\
\text { Length }(l) \text { of the } \\
\text { combustor(m) }\end{array}$ & $\begin{array}{c}P_{1} \\
\text { (US of } \\
\text { swirler) }\end{array}$ & $\begin{array}{c}P_{2} \\
\text { (DS of } \\
\text { the swirler) }\end{array}$ & $\begin{array}{c}P_{3} \\
\text { (DS of } \\
\text { the swirler) }\end{array}$ & $\begin{array}{c}T \\
\text { (DS of } \\
\text { swirler) }\end{array}$ \\
\hline 0.7 & $60 \mathrm{~mm}$ & $0.45 \mathrm{~m}$ & $0.65 \mathrm{~m}$ & $0.45 \mathrm{~m}$ \\
0.8 & $60 \mathrm{~mm}$ & $0.45 \mathrm{~m}$ & $0.75 \mathrm{~m}$ & $0.45 \mathrm{~m}$ \\
1.0 & $60 \mathrm{~mm}$ & $0.45 \mathrm{~m}$ & $0.95 \mathrm{~m}$ & $0.45 \mathrm{~m}$ \\
\hline
\end{tabular}

A 16 bit analog to digital converter (NI-9215) having a resolution and input voltage range of $0.305 \mathrm{mV}$ and $\pm 10 \mathrm{~V}$ is used to collect data fromthe transducers. The collected data is monitored on signal express LAB view software. The data are collected in text files, which are then analysed in MATLAB. $l$ and $l_{\text {mix }}$ are varied for different conditions of the experiment with $A R$.

\section{Results and Discussion}

Experiments are performed in the following conditions. Inlet air supply temperature is measured as $305 \mathrm{~K}$. Air flow rate $(V)$ is varied between $600-1700$ slpm (standard litres per minute) in steps of 100 slpm, while fuel flow rate $(V)$ is altered in the range $13-44 \mathrm{slpm}$, stepping at $3 \mathrm{slpm}$. Their flow rates are increased simultaneously, and the equivalence ratio ( $\phi$ ) lies between $0.63-0.73$. 
Figure 3 shows the variation of equivalence ratio and steady-state temperature inside the combustor with fuel flow rate and airflow rate. With increase in airflow rate, we observe an increase in equivalence ratio, which results in increase in steady state temperature. Table 2 gives the details about different airflow rate, fuel flow rate with the residence time for different $l_{m i x}$.

Table 2: Airflow rate, fuel flow rate corresponding equivalence ratio with residence time for airflow rate and injection location

$\tau_{40 \mathrm{~mm}}, \tau_{80 \mathrm{~mm}}$, are the residence times for 40 and $80 \mathrm{~mm}$ injection at different airflow rates calculated mathematically.

\begin{tabular}{c|c|c|c|c}
\hline$V_{\alpha}$ & $V_{f}$ & $\phi$ & $\begin{array}{c}\tau_{40 \mathrm{~mm}} \\
(\mathrm{Sec})\end{array}$ & $\begin{array}{c}\tau_{80 \mathrm{~mm}} \\
(\mathrm{Sec})\end{array}$ \\
\hline 600 & 13 & 0.64 & 0.0030 & 0.0060 \\
700 & 16 & 0.66 & 0.0025 & 0.0051 \\
800 & 19 & 0.67 & 0.0022 & 0.0045 \\
900 & 22 & 0.68 & 0.0020 & 0.0040 \\
1000 & 24 & 0.69 & 0.0018 & 0.0036 \\
1100 & 27 & 0.70 & 0.0016 & 0.0032 \\
1200 & 30 & 0.71 & 0.0015 & 0.0030 \\
1300 & 33 & 0.71 & 0.0013 & 0.0027 \\
1400 & 35 & 0.71 & 0.0012 & 0.0025 \\
1500 & 38 & 0.72 & 0.0012 & 0.0024 \\
1600 & 41 & 0.72 & 0.0011 & 0.0022 \\
1700 & 44 & 0.73 & 0.0010 & 0.0021 \\
\hline
\end{tabular}

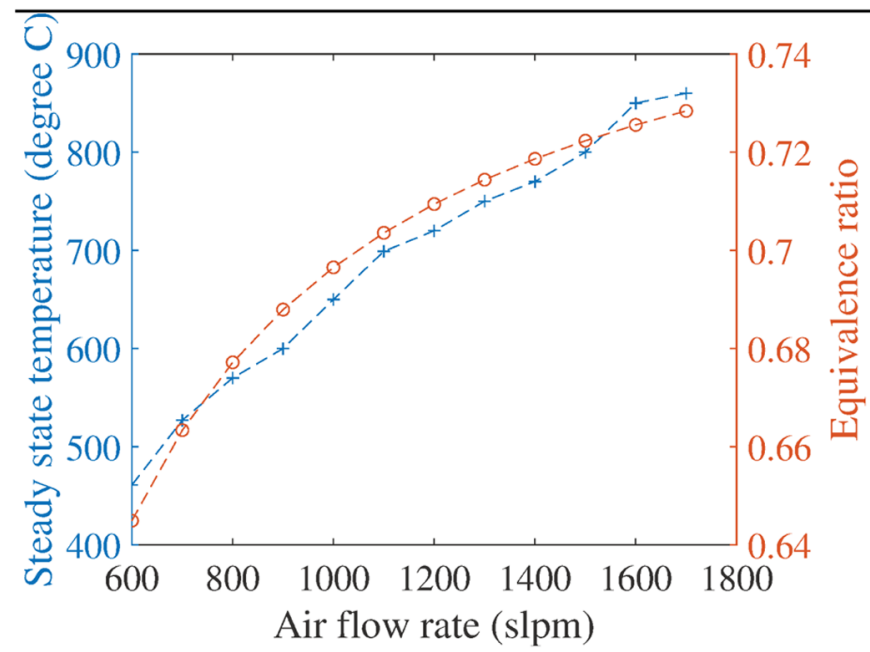

Figure 3:Steady-state temperature and equivalence ratio variation with airflow rate, steady-state temperature shown in ' + ' and equivalence ratio shown in 'o'

Results are presented for three total lengths of the combustor $(l=1$, $0.8,0.7 \mathrm{~m}$ ) and two locations of fuel injection measured from the face of the swirler $\left(l_{\text {mix }}=40,80 \mathrm{~mm}\right)$ at two area ratios 1 and 0.12 . The choice of $A R=0.12$ is as follows. Since the nozzle is compact compared to the total length $(l)$ of the combustor, an analytical expression for the acoustic reflection coefficient (Murugesan et al. 2018) can be adapted to obtain the exit reflection coefficient $R$ for various $A R$ (refer Appendix A).

The reflection coefficient is defined as the ratio of the amplitude of the reflected wave to the incident wave[21]. In the present study, the reflection coefficient at the exit $R$ is expressed, and the final expression and the related derivation are reported in appendix A and from equation A12.

$$
R_{p}=\frac{\tilde{g}_{n o}}{\tilde{f}_{n o}}
$$

The reflection coefficient in terms of impedance is expressed as (Kinsler et al. 1999):

$$
R=\frac{z-1}{z+1}
$$

Where, $\mathrm{z}$ is the impedance, which is the ratio of acoustic pressure at the surface to the volume velocity at the surface. This is expressed as (Kinsler et al. 1999):

$$
z=\frac{\hat{p}}{\hat{u}}
$$
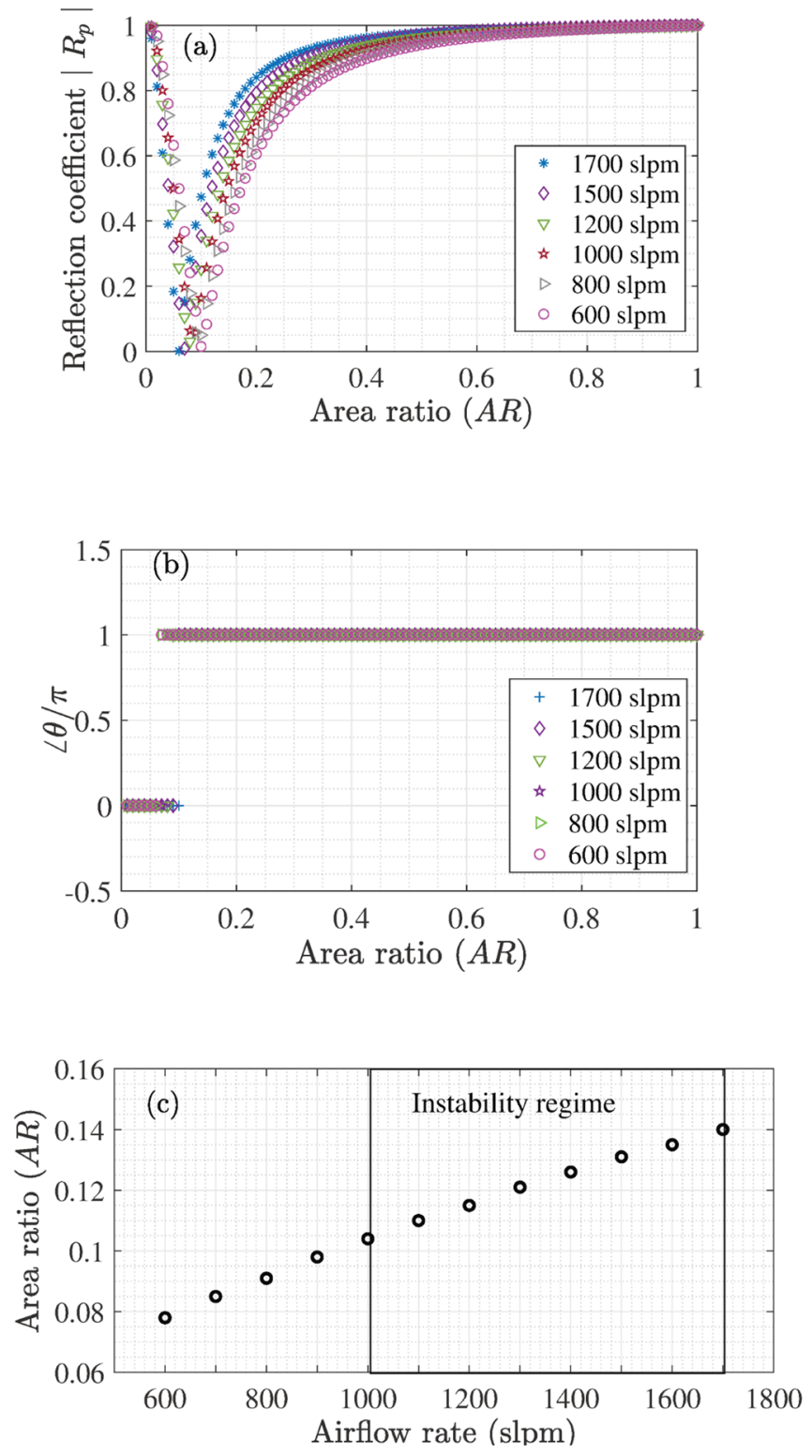

Figure 4:

(a) Variation of the exit reflection coefficient $R$ with area ratio $(A R)$ (b) Variation of phase $\left({ }^{\angle \theta} / I_{\pi}\right)$ for different airflow rate with area ratio $(A R)$ (c) $A R$ Corresponding to $R_{p}=0$ at different airflow rates.

In the present study, the reflection coefficient changes as the area ratio changes at the exit of the duct, this can be concluded as:

$$
\text { At } A R=1 \text { (open end), } \hat{p}=0 \text {, indicating } z=0 \text { and } R=-1
$$$$
\text { At } A R=0 \text { (close end), } \hat{u}=0 \text {, indicating } z=\infty \text { and } R=+1
$$ 
Figure 4(a) and (b), represents the variation of $R_{n}$ and $\angle \theta / \pi$ with $A R$ for various airflow rates. For $A R=1, \angle \theta I_{\pi}=1$ indicating acoustically open exit. As the $A R$ is reduced, $\left|R_{p}\right|$ converges to zeros (anechoic boundary) with phase $(\angle \theta /)$ instantly changing from 1 to 0 . Further decreasing $A R$, the $\left|R_{p}\right|$ increases to 1 with $\angle \theta / \pi$ equals 0 representing an acoustically closed exit. In the intermediate region $\left|R_{p}\right|$ reduces to zero (anechoic boundary) and the $\angle \theta / \pi$ changes from 1 to 0 : the exact location of $A R$ for $\left|R_{p}\right|=0$, increases with the airflow rate. Figure 4(c) shows the variation of $A R$ at which $\left|R_{p}\right|=0$, with the airflow rate. This critical $A R$ lies between $0.08-0.14$ for the operating regime, where instability is observed in the range of $1000-1700 \mathrm{~s} 1 \mathrm{pm} .\left|R_{p}\right|$ is calculated theoretically from the derivation used in appendix $\mathrm{A} ;{ }^{p}$ on the contrary, the instability regime has been determined from the experimental observation. We choose to fix $A R$ $=0.12$, so that exit is close to anechoic and ITA driven instability is expected to occur for the flow rates encountered.From figure 4(a), the reflection coefficient at the exit is not only the function of $A R$ but also the airflow rate inside the combustor, which changes the reflection coefficient value at higher flow rate.Experiments are performed twice for each case, and the deviation in dominant frequency is found to be less than $8 \mathrm{~Hz}$.

Figure 5 shows the time series data for pressure fluctuations, measured at $p_{2}$ for $V \alpha=600,1000$ and $1700 \mathrm{slpm}$ at $A R=1, l=1 \mathrm{~m}$ at $l_{\text {mix }}=40 \mathrm{~mm}$. The first and second columns correspond to the raw signals measured during the entire duration (4s) and zoomed-in view of the oscillations, respectively. Power spectral density (PSD), extracted from the signal using the Welch method (Hall and River 2009) in MATLAB is shown in the third column.In Figure 5, at the lowest airflow rate, the signal consists of combustion noise and a shallow peak at $130 \mathrm{~Hz}$. As the airflow rate is increased, PSD becomes sharper at definite peaks, indicating a transition to combustion instability. The dominant frequency is observed to be 130 , 169 , and $203 \mathrm{~Hz}$ in figure 5 . The second peak values are almost twice of dominant frequency, i.e., 264,334 , and $408 \mathrm{~Hz}$.In the further discussion, the term frequency indicates dominant frequency, unless specified.

In the present experiment, we observe instability regime in the range of 1000-1700slpm of airflow rate. The results are discussed only in this particular instability regime. In subsequent sections, the variation of frequency and mode shape is analysed for the parameters, $V_{\alpha^{\prime}}, l, l_{\text {mix }}$ and $A R$.

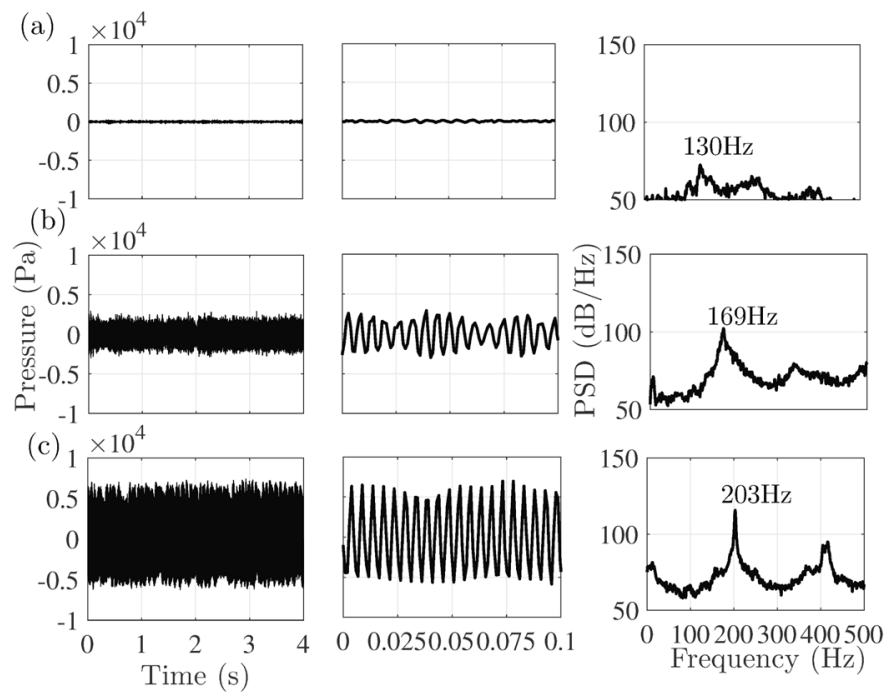

Figure 5: Time series (column $1 \& 2$ ) and power spectral density (column 3) of pressure fluctuations at $P_{2}$ for (a) $V_{f}=10 \mathrm{slpm}, V_{\alpha}=600 \mathrm{slpm}$, (b) $=18 \mathrm{slpm},=1000 \mathrm{slpm}$ and (c) $=32 \mathrm{slpm},=1700 \mathrm{slpm}$ respectively.

\subsection{Variation of the frequency with? $V_{\alpha}, l, l$ mix, and $A R$}

For $\mathrm{AR}=1$, figure $6 \mathrm{a}, \mathrm{c}$ shows the variation of frequency with airflow rate for the three lengths 1 , at different $l_{\text {mix }}$. As 1 is decreased from $1 \mathrm{~m}$ to $0.7 \mathrm{~m}$, the frequency is observed to increase. On the other hand, the frequency remains fairly constant with $l_{\text {mix }}$ (compare figure $6 \mathrm{a} \& \mathrm{c}$ ). This behaviour indicates that the mechanism of instability strongly depends on the feedback loop associated with 1 . Along with the fact that $|R p|=1$, $\angle \theta / I_{\pi}=1$ for $A R=1$, the instability can be attributed to the combustor acoustic field (acoustic-driven instability). As the airflow rate increases, the steady-state temperature downstream of the flame increases, shown in figure 3, which leads to an increase in the frequency. In the case of $A R=$ 0.12 (figure $6 \mathrm{~b}, \mathrm{~d}$ ), we observe that the frequency is lower compared to $A R=1$. Figure $6 \mathrm{~b}(l=80 \mathrm{~mm})$ shows that the dominant frequency is a weak function of the length $l$, while the dependence is strong with the location of fuel injection $l_{\text {mix }}$ (compare figure $6 \mathrm{~b} \& \mathrm{~d}$ ). Hence for $A R=$ 0.12 , the mechanism should involve a feedback loop containing the distance $\left(l_{\text {mix }}\right)$ between the location of fuel injection and flame (flame is observed to be anchored to the swirler). With the use of network models, it was shown in many previous investigation [16] (Emmert et al. 2015) (Hoeijmakers et al. 2014) that the ITA feedback loop is responsible for the occurrence of instability. The feedback loop is formed between velocity fluctuations in the airflow at the location of fuel injection and the flame heat release rate. Velocity fluctuations cause local equivalence ratio fluctuations at fuel injection location. These fluctuations are transported with the local convective flow velocity and perturb the heat release rate (Bomberg, Emmert, and Polifke 2015) (Hosseini et al. 2018).The associated time lag is approximately the time taken by the flow to convect the distance $l_{\text {mix }}$ which is presented in table 2 . The frequency of the ITA mode is inversely proportional to the convective time lag (refer table 2 for residence time) in the flame transfer function (Albayrak et al. 2017) Therefore for a given $l_{\text {mix }}$, the frequency of ITA mode increases with airflow rate (figure $6 \mathrm{~b} \& \mathrm{~d}$ ). For the mechanism described above, the mean equivalence ratio does not play a role. Hence, we expect equivalence ratio variations $(0.63-0.73)$ has no or little effects on the ITA frequency observed in the experiments. By decreasing $l_{\text {mix }}$ from 80 to $40 \mathrm{~mm}$, it is expected that the dominant frequency should increase. However, a decrease is observed between figure $6 \mathrm{~b} \& \mathrm{~d}$. This indicates that the time lag is no longer related or restricted to the mechanism associated with equivalence ratio fluctuations. A more definite conclusion in this regard can be made with the measurement of the flame transfer function. A second important difference observed between figure $6 \mathrm{~b} \& \mathrm{~d}$ is that the instability at $l_{\text {mix }}=$ $40 \mathrm{~mm}$, shows a dependence on the length of the combustor $(l)$.

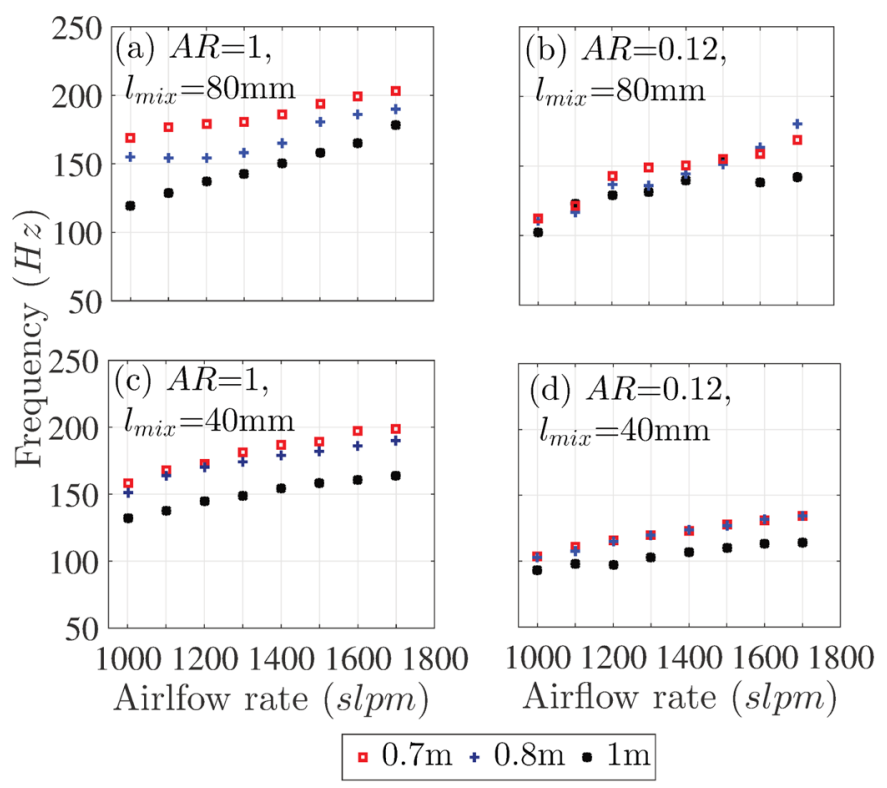

Figure 6:Variation of the dominant frequency with airflow rate forvarious combinations of $l, l_{m i x}$, and $A R$.

The frequency associated with $l=1 \mathrm{~m}$ is consistently lower than for $l=$ $0.8,0.7 \mathrm{~m}$. It is possible that there is feedback due to acoustic-driven instability playing a role for the instability at $l=1 \mathrm{~m}$. We observe from figure 6 (d) that the length of the combustor does not affect the frequency associated with $l=0.7$ and $0.8 \mathrm{~m}$. However, the corresponding frequency is always higher than the case of $1 \mathrm{~m}$ long configuration. Therefore, it can be concluded that for $l=1 \mathrm{~m}$ at $l_{\text {mix }}=40 \mathrm{~mm}$, the eigenmode has the characteristic of acoustic mode, while for $1=0.7$ and $0.8 \mathrm{~m}$ at $l=40$ $\mathrm{mm}$, it has the character of ITA mode. Emmert et al. (Emmert et al. 2017) also stated that for determining the stability of combustion system both acoustic mode and ITA mode should be considered. It appears that for figure 6(d) the mode of instability switches from ITA to acoustic with change in the length of combustor at reduced reflection coefficient. Further the comparison between two $l_{\text {mix }}$ is presented in figure 7 . 


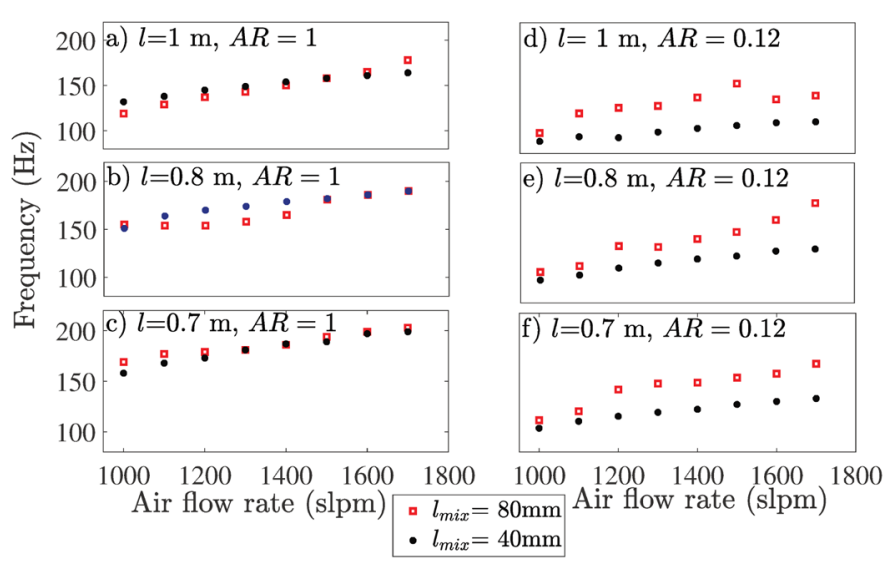

Figure 7: Variation of the dominant frequency with airflow rate for various combinations of $l, l_{m i x}$, and $A R$.

Figure 7, shows the variation of frequency with airflow rate comparing different $l$ for fixed $A R$ and 1. From the figure 7 (a), (b) and (c), for the length of $0.8 \mathrm{~m}$ and $0.7 \mathrm{~m}$ variation in $l_{\text {mix }}$ of 40 and $80 \mathrm{~mm}$ the frequency varies between $150-210 \mathrm{~Hz}$. For $1 \mathrm{~m}$ length the frequency varies between $120-180 \mathrm{~Hz}$ for $l_{\text {mix }}$ of 40 and $80 \mathrm{~mm}$. The observation clearly gives a strong support for the earlier statement that at lower acoustic loss (Open end $/ A R=1$ ) the frequency is strongly depended on 1 . The $l$ dependency is minimal which signifies the acoustic mode inside the combustor. For figure 7 (d), (e) and (f), for $l=1,0.8$, and $0.7 \mathrm{~m}$ length, the frequency variation is between $80-160 \mathrm{~Hz}$. The effect of $l_{\text {mix }}$ is higher in case of higher acoustic losses with $A R=0.12$ (partially close end) which support the earlier statement that the instability is strong function of $l_{\text {mix }}$ which is true for ITA driven instability. The $80 \mathrm{~mm}$ injection location shows higher value of frequency compared to $40 \mathrm{~mm}$. The difference in frequency increases with airflow rate. With increase in airflow rate the velocity of flow increases. From the earlier investigation (Albayrak et al. 2017) the bulk flow velocity plays a crucial role on ITA mode of instability. The bulk flow velocity is directly related to convection time of mixture inside the mixing tube. It is observed that as the exit boundary condition tends to anechoic, it leads to onset of ITA mode of instability in the system. It is also clear that $l$ plays a very crucial role in identifying the frequency; with higher the value of $l_{\text {mix }}$, the higher the frequencies are observed. $l_{\text {mix }}$ can also be connected to the mixing of fuel and air inside the mixing tube which can be further expressed in terms of degree of mixing. Further investigation is required to express the $l_{\text {mix }}$ in degree of mixing.

Figure 8 shows the variation of frequency with airflow rate comparing different $A R$ with fixed $l_{\text {mix }}$ and $l$ values. From figure 8 , the $A R=0.12$ shows lesser value of frequency compared to $A R=1$ in all the cases. However, the difference is found to be less for $l_{m i x}=80 \mathrm{~mm}$ and $l=1 \mathrm{~m}$. The lesser value of frequency for $A R=0.12$ can be considered to be the cause

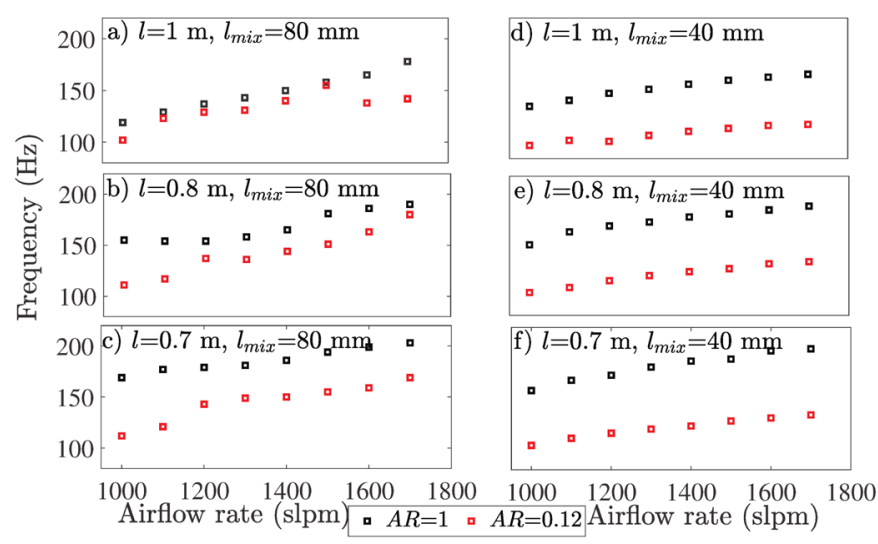

Figure 8:Variation of the dominant frequency with airflow rate for various combinations of $l, l_{m i x}$, and $A R$. of end correction. The air at the end of pipe takes part in acoustic oscillation which makes the end of pipe appear to be acoustically somewhat longer than its physicallength. The apparent length of the pipe is considered as the length correction (Johan Liljencrants 2006). In the case of $A R=0.12$ the correction length of tube is longer than $A R=1$, which implies the frequency value for $A R=1$ is higher compared to $A R=0.12$.

\section{2 Variation of mode shape with $V_{\alpha}, l, l_{m i x}$, and $A R$}

Figure 9 shows the variation of the phase difference between pressure fluctuations measured near the exit of the combustor $\left(p_{3}\right)$ and fuel injection location $(p$,$) at the dominant frequency. This phase difference is obtained$ through the complex Fourier transform of thetime series signal at

$$
p_{1}=\mathfrak{R}\left(\hat{p}_{1} \exp (i \omega t)\right) \text { and } p_{3}=\mathfrak{R}\left(\hat{p}_{3} \exp (i \omega t)\right) . \wedge
$$

symbol indicates complex Fourier transform, $i \sqrt{ }-1$, and $\omega$ is the circular frequency. For both the exit area ratios, we observe the phase difference to be negative and decreasing with the airflow rate. This can be explained as follows: For $A R=1$, the theoretical value of $\left|R_{p}\right|=1, \angle \theta /=1$ indicating no loss of acoustic energy at the exit. However, in reality, losses do exist. Hence a perfect standing wave pattern does not occur. Since acoustic energy leaves the system, one expects a right running wave component in the mode shape. Inside the combustor, the presence of flame and area jump at the swirler does not contribute to the phase shift in pressure fluctuations. Hence, a negative value of the phase difference between $\left(p_{3}\right)$ and $\left(p_{t}\right)$ indeed indicates the presence of the right running wave. As the airflow rate is increased, more acoustic energy is lost through the mean flow, indicating more contribution from the right running wave. Therefore, the phase difference decreases further with the airflow rate.

For $A R=0.12$, the value of $\left|R_{p}\right|$ is reduced (refer Figure 4a), which indicates more acoustic losses than for $A R=1$. Higher acoustic losses lead to more contribution (in comparison to that for $\mathrm{AR}=1$ ) from the right running wave. This reduces further the phase difference between $p_{3}$ and $p_{i}$ $p_{1}$. Therefore, the phase difference for $\mathrm{AR}=0.12$ is always lower than that for $A R=1$ (compare cross and plus symbols in figure 9). As the airflow rate is increased, more acoustic energy is lost through the mean flow, indicating more contribution from the right running wave. Therefore, we expect the phase difference decreases with the airflow rate. However, from the experimental results (Figure $9 \mathrm{~d} \& \mathrm{f}$ ), we observe that the phase difference does not strictly decrease with the airflow rate. The reason will be explored in our further investigations.
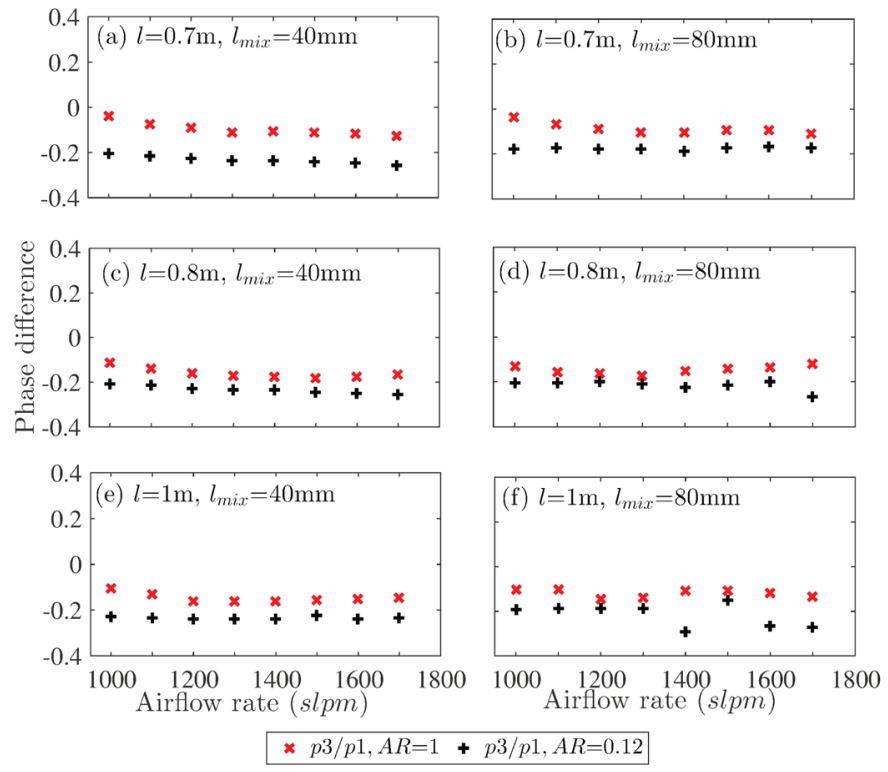

Figure 9: Phase difference between $p_{3}$ and $p_{1}$ for various combinations of $1,1_{\text {mix }}$, and AR.

\section{Conclusions}

The present study focuses on understanding intrinsic thermo acoustic (ITA) feedback-driven instability in a range of parameters: exit area ratio, total combustor chamber length, and location of fuel injection. Experiments are performed in a swirl stabilized partially premixed turbulent combustor at two end conditions $(A R=1 \& 0.12)$.Unsteady 
pressure measurements made at three locations are used for the analysis. Reducing the exit area increases acoustic losses and results in changing the mechanism of instability. For $A R=1$, acoustic-driven instability is dominant, and the frequency is a strong function of the total length of the combustor. On the other hand, the ITA feedback loop drives in stability for $A R=0.12$. The dominant frequency is almost solely dependent on the time lag associated with the flame transfer function. The time lag is dictated by the location of the fuel injection(Emmert et al. 2017).

For $A R=0.12$, the effect of $l$ on frequency is dominant, as compared to the case $A R=1$. For $A R=0.12$ with increase in airflow rate the frequency difference between two $l_{\text {mix }}$ increases, which indicates the effect of the bulk velocity on ITA mode of instability.

The frequency value for $A R=1$ is higher compared to $A R=0.12$ due to end correction length effect.

The phase difference between pressure fluctuations near the exit of the combustor and fuel location indicates the presence of the right running wave component in the mode shape. The phase difference is consistently lower for $A R=0.12$ (in comparison to $A R=1$ ), indicating more contribution of the right running wave, arising due to increased acoustic losses

In real system for determining the stability of combustor, the acoustic and ITA mode both must be considered. The boundary condition in real gas turbine changes frequently leads to change in reflection coefficient at the exit which changes the mode of instability inside the combustor.

Furthermore, the measurement of the reflection coefficient at boundary and flame dynamics is an important factor for a detailed study of the mechanism involved in instability. The present study is only limited to the variation of different parameters and its effect on the instability mechanism, which is dictated in the form of dominant frequency and the phase difference. A more and wide range of studies is required for the detailed study of instability mechanism, which includes the determination of flame transfer function, degree of premixing for different injection locations and determining the reflection coefficient at the boundaries of the experimental setup.

\section{Acknowledgement:}

This work was supported by Gas Turbine Research Establishment, India under GATET scheme (project number:GTRE/AE/2015253)

Appendix A. Reflection coefficients using compact nozzle formulation

Figure 2 shows the diagram of the compact nozzle with boundary conditions imposed for the present study. Reflection coefficient for pressure $\left(R_{p}\right)$ is used to describe theboundary for the incident acoustic waves. In the limit of a compactnozzle, one can obtain an analytical expression for the above coefficients. Acousticpressure/velocity fluctuations are decomposed in terms of right $\left(\widetilde{f}_{n}\right)$ and left $\left(\widetilde{g}_{n}\right)$ running waves. The relations are as follows.

$$
\frac{\overline{p_{n}^{\prime}}}{\left(\overline{\left.\widetilde{\rho_{n}} \widetilde{c_{n}}\right)}\right.}=\widetilde{f_{n}}+\widetilde{g_{n}}, u_{n}=\widetilde{f_{n}}-\widetilde{g_{n}}(\mathrm{~A} 1)
$$

Where $p_{n}$ and $\left(c_{n}\right)$ represent the steady-state density and speed of sound, respectively. The entrance and exit of the nozzle are indicated by the subscript $\mathrm{n}$ taking no and $\mathrm{e}$, respectively.

Note that the exit of the nozzle (location $x=x_{e}=1$ ) is open to the ambient atmosphere. The aim is to obtain the reflection coefficients for the pressure $\left(R_{p}\right)$ at the entrance to the nozzle, $x_{n 0}$. In the limit of the compact nozzle, the areaand the associated flow property jumps between locations $x_{n o}$ and $x=1$. Expressionsfor the right $\left(\widetilde{f}_{n o}\right)$ and left $\left(\widetilde{g}_{e}\right)$ running waves at locations $x_{n o}$ and $x_{e}$, respectively, are shown below.

$$
\begin{gathered}
\frac{\widetilde{g_{n o}}}{\widetilde{c_{n o}}}=R_{g_{n o} f_{n o}} \frac{\widetilde{f_{n o}}}{\widetilde{c_{n o}}}+R_{g_{n o} g_{e}} \frac{\widetilde{g_{e}}}{\widetilde{\widetilde{c_{e}}}} \\
\frac{\widetilde{f_{e}}}{\widetilde{\tau_{e}}}=R_{f_{e} f_{n o}} \frac{\widetilde{f_{n o}}}{\widetilde{c_{n o}}}+R_{f_{e} g_{e}} \frac{\widetilde{g_{e}}}{\widetilde{c_{e}}}
\end{gathered}
$$

The above two equations are written based on the following fact. The left running acoustic wave $\left(\widetilde{g}_{\text {mo }}\right)$ is generated due to (i) the reflection of the right running wave $(\widetilde{f})$ ? atstation xno, and (ii) the transmission of the left running wave $(\widetilde{g})$ through the nozzle since thenozzle is not choked for the operating conditions at station xno. Similarly, the right running acoustic wave $\left(\widetilde{f_{n}}\right)$ ?occurs due to (i) the transmission of the right running wave $(\widetilde{f})$ through the unchoked nozzle, (ii) thereflection of the left running wave $\left(\widetilde{g_{e}}\right)$ at station xe.
The coefficients $R_{\text {gno frno }}, R_{\text {gno ge }}, R_{\text {fe fno }}$, and $R_{\text {fe ge }}$ represent the pressure reflection coefficient at location $x_{n}$, pressure transmission coefficient from location xe to $x_{n o}$, pressure transmission coefficient from location $x_{n o}$ to $x$, the inverse of the pressure reflection coefficient at location $x$ respectively. The coefficients can be obtained by manipulating the expressions for the acoustic/transmission coefficients for compact subcritical nozzle given in Marble \& Candel (1977)

$$
\begin{gathered}
R_{g_{n o} f_{n o}}=\left(\frac{M_{e}-M_{n o}}{1-M_{n o}}\right)\left(\frac{1+M_{n o}}{M_{n o}+M_{e}}\right)\left(\frac{1-(\gamma-1) M_{n o} M_{e} / 2}{1+(\gamma-1) M_{n o} M_{e} / 2}\right) \\
R_{f_{e} f_{n o}}=\left(\frac{M 2_{e}}{1+M_{e}}\right)\left(\frac{1+M_{n o}}{M_{n o}+M_{e}}\right)\left(\frac{1+(\gamma-1) M_{e}^{2} / 2}{1+(\gamma-1) M_{n o} M_{e} / 2}\right) \\
R_{g_{n o} g_{e}}=\left(\frac{2 M_{n o}}{M_{n o}+M_{e}}\right)\left(\frac{1-M_{e}}{1-M_{n o}}\right)\left(\frac{1-(\gamma-1) M_{n o}^{2} / 2}{1+(\gamma-1) M_{n o} M_{e} / 2}\right) \\
R_{f_{e} g_{e}}=\left(\frac{M_{e}-M_{n o}}{1-M_{n o}}\right)\left(\frac{1-M_{e}}{M_{n o}+M_{e}}\right)\left(\frac{1-(\gamma-1) M_{n o} M_{e} / 2}{1+(\gamma-1) M_{n o} M_{e} / 2}\right)
\end{gathered}
$$

Since location $x$ represents the atmosphere, we apply an acoustically open boundarycondition, $\widetilde{p}_{e}=0 \rightarrow \widetilde{f}_{e}+\widetilde{g}_{e}=0$. Using this relation, $\widetilde{g}_{e}$ is eliminated from equation A10 to obtain

$$
\begin{aligned}
& \frac{\widetilde{g_{n o}}}{\widetilde{c_{n o}}}=R_{g_{n o} f_{n o}} \frac{\widetilde{g_{n o}}}{\widetilde{c_{n o}}}+R_{g_{n o} g_{e}} \frac{\widetilde{f_{e}}}{\widetilde{c_{e}}} \\
& \frac{\widetilde{f_{e}}}{\widetilde{c_{e}}}=R_{f_{e} f_{n o}} \frac{\widetilde{f_{n o}}}{\widetilde{c_{n o}}}-R_{f_{e}} g_{e} \frac{\widetilde{f_{e}}}{\widetilde{c_{e}}}
\end{aligned}
$$

Using the above two equations, we eliminate $\tilde{f}_{e}$ to obtain

$$
\tilde{g}_{n o}=R_{p} \tilde{f}_{n o}
$$

Where,

$$
R_{p}=R_{g_{n o} f_{n o}}-\frac{R_{g_{n o} g_{e}} R_{f_{e} f_{n o}}}{1+R_{f_{e}} g_{e}}
$$

From the form of equation $\mathrm{A} 12$, we observe that $R_{p}$ are the reflection coefficients associated with incident pressure waves at $x_{\text {pro }}$. The analytical expressions for $R_{p}$ are used to obtain the continuous curves in figure $2 \mathrm{a}$.

\section{References}

Albayrak, Alp, Thomas Steinbacher, Thomas Komarek, and Wolfgang Polifke. 2017. "Convective Scaling of Intrinsic Thermo-Acoustic Eigen frequencies of a Premixed Swirl Combustor." Proceedings of ASME Turbo Expo 140(April):1-11.

Armitage, C. A., R. Balachandran, E. Mastorakos, and R. S. Cant. 2006. "Investigation of the Nonlinear Response of Turbulent Premixed Flames to Imposed Inlet Velocity Oscillations." Combustion and Flame.

Bomberg, S., T. Emmert, and W. Polifke. 2015. "Thermal versus Acoustic Response of Velocity Sensitive Premixed Flames." Proceedings of the Combustion Institute 35(3):3185-92.

Chen, Yong, Lorna J. Ayton, and Dan Zhao. 2019. "Modelling of Intrinsic Thermoacoustic Instability of Premixed Flame in Combustors with Changes in Cross Section." Combustion Science and Technology 00(00):1-20.

Courtine, Emilien et al. 2014. "Causality and Intrinsic Thermoacoustic Instability Modes." OATAO 2014(July):169-78.

Courtine, Emilien, Laurent Selle, and Thierry Poinsot. 2015. "DNS of Intrinsic ThermoAcoustic Modes in Laminar Premixed Flames." Combustion and Flame 162(11):4331-41.

Duran, I. et al. 2014. "In Modern Aero-Engines." (7):1-11.

Durox, D., T. Schuller, N. Noiray, and S. Candel. 2009. "Experimental Analysis of Nonlinear Flame Transfer Functions for Different Flame Geometries." Proceeding. of the Combustion Institute 32 I(1):1391-98.

Emmert, T., S. Bomberg, S. Jaensch, and W. Polifke. 2017. "Acoustic and Intrinsic Thermoacoustic Modes of a Premixed Combustor." Proceedings of the Combustion Institute 36(3):3835-42. 
Emmert, Thomas, Sebastian Bomberg, and Wolfgang Polifke. 2015. "Intrinsic Thermoacoustic Instability of Premixed Flames." Combustion and Flame 162(1):75-85.

Hall, Prentice and Upper Saddle River. 2009. Spectral Analysis of Signals.

Hase, Koji and Y. Kori. 1996. "Effect of Premixing NO , Formation of Fuel Gas and Air On." Fuel 75(13):1509-14.

Hoeijmakers, Maarten, Viktor Kornilov, Ines Lopez Arteaga, Philip de Goey, and Henk Nijmeijer. 2016. "Flame Dominated Thermoacoustic Instabilities in a System with High Acoustic Losses." Combustion and Flame 169:209-15.

Hoeijmakers, Maarten, Viktor Kornilov, Ines Lopez Arteaga, Philip de Goey, and Henk Nijmeijer. 2014. "Intrinsic Instability of Flame-Acoustic Coupling." Combustion and Flame 161(11):2860-67.

Hoeijmakers, Maarten, Viktor Kornilov, Ines Lopez Arteaga, Philip De Goey, and Henk Nijmeijer. 2015. "Flames in Context of Thermo-Acoustic Stability Bounds." Proceedings of the Combustion Institute 35(1):1073-78.

Hosseini, N. et al. 2018. "Intrinsic Thermoacoustic Modes and Their Interplay with Acoustic Modes in a Rijke Burner." International Journal of Spray and Combustion Dynamics 10(4):315-25.

Johan Liljencrants. 2006. "End Correction at Flue Pipe Mouth." 1.

Kinsler, Lawrence E., Austin R. Frey, Alan B. Coppens, and James V Sanders. 1999. "Fundamentals of Acoustics." Fundamentals of Acoustics, 4th Edition, by Lawrence E. Kinsler, Austin R. Frey, Alan B. Coppens, James V. Sanders, Pp. 560. ISBN 0-471-84789-5. Wiley-VCH, December 1999. 1:560.

Marble, F. E. and S. M. Candel. 1977. "Acoustic Disturbance from Gas Non-Uniformities Convected through a Nozzle." Journal of Sound and Vibration 55(2):225-43.

Morgans, Aimee S. and Ignacio Duran. 2016. "Entropy Noise: A Review of Theory, Progress and Challenges." International Journal of Spray and Combustion Dynamics 8(4):285-98.
Morgans, Aimee S., Chee Su Goh, and Jeremy A. Dahan. 2013. "The Dissipation and Shear Dispersion of Entropy Waves in Combustor Thermoacoustics." Journal of Fluid Mechanics 733:R2

Mukherjee, Nalini Kanta and Victor Shrira. 2017. "Intrinsic Flame Instabilities in Combustors: Analytic Description of a 1-D Resonator Model." Combustion and Flame 185:188-209.

Murugesan, Meenatchidevi, Balasubramanian Singaravelu, Abhijit K. Kushwaha, and Sathesh Mariappan. 2018. "Onset of Flame-Intrinsic Thermoacoustic Instabilities in Partially Premixed Turbulent Combustors." International Journal of Spray and Combustion Dynamics 10(3):171-84.

Silva, Camilo F., Malte Merk, Thomas Komarek, and Wolfgang Polifke. 2017. "The Contribution of Intrinsic Thermoacoustic Feedback to Combustion Noise an Resonances of a Confined Turbulent Premixed Flame." Combustion and Flame 182:269-78

Stopper, Ulrich et al. 2013. "Experimental Study of Industrial Gas Turbine Flames Including Quantification of Pressure Influence on Flow Field, Fuel/Air Premixing and Flame Shape." Combustion and Flame 160(10):2103-18.

Strutt, John William and Baron Rayleigh. 1877. "The Theory of Sound." Volume 1.

Tay-Wo-Chong, Luis, Sebastian Bomberg, Ahtsham Ulhaq, Thomas Komarek, and Wolfgang Polifke. 2011. "Comparative Validation Study on Identification of Premixed Flame Transfer Function." Proceedings of the ASME Turbo Expo 2(PARTS A AND B):1109-18.

Tim Lieuwen. 2003. "Combustion Driven Oscillations." Gas Turbine 16-18.

Venkataraman, K. K. et al. 1999. "Mechanism of Combustion Instability in a Lean Premixed Dump Combustor." Journal of Propulsion and Power 15(6):909-18.

Williams, Forman A. 1985. Combustion Theory. Second edi. Princeton University: CRC press 